\title{
Choosing $\mathrm{k}$ from $\mathrm{m}$ : Feasible elimination procedures reconsidered
}

Citation for published version (APA):

Peleg, B., \& Peters, H. (2017). Choosing k from m: Feasible elimination procedures reconsidered. Games and Economic Behavior, 103, 254-261. https://doi.org/10.1016/j.geb.2015.12.008

Document status and date:

Published: 01/05/2017

DOI:

10.1016/j.geb.2015.12.008

Document Version:

Publisher's PDF, also known as Version of record

Document license:

Taverne

Please check the document version of this publication:

- A submitted manuscript is the version of the article upon submission and before peer-review. There can be important differences between the submitted version and the official published version of record.

People interested in the research are advised to contact the author for the final version of the publication, or visit the DOI to the publisher's website.

- The final author version and the galley proof are versions of the publication after peer review.

- The final published version features the final layout of the paper including the volume, issue and page numbers.

Link to publication

\footnotetext{
General rights rights.

- You may freely distribute the URL identifying the publication in the public portal. please follow below link for the End User Agreement:

www.umlib.nl/taverne-license

Take down policy

If you believe that this document breaches copyright please contact us at:

repository@maastrichtuniversity.nl

providing details and we will investigate your claim.
}

Copyright and moral rights for the publications made accessible in the public portal are retained by the authors and/or other copyright owners and it is a condition of accessing publications that users recognise and abide by the legal requirements associated with these

- Users may download and print one copy of any publication from the public portal for the purpose of private study or research.

- You may not further distribute the material or use it for any profit-making activity or commercial gain

If the publication is distributed under the terms of Article $25 \mathrm{fa}$ of the Dutch Copyright Act, indicated by the "Taverne" license above, 


\title{
Choosing $k$ from $m$ : Feasible elimination procedures reconsidered
}

\author{
Bezalel Peleg ${ }^{\mathrm{a}}$, Hans Peters ${ }^{\mathrm{b}, *}$ \\ a The Federmann Center for the Study of Rationality and the Institute of Mathematics, The Hebrew University of Jerusalem, Jerusalem 91904, \\ Israel \\ ${ }^{\mathrm{b}}$ Department of Quantitative Economics, Maastricht University, Maastricht, The Netherlands
}

\section{A R T I C L E I N F O}

\section{Article history:}

Received 9 March 2015

Available online 8 January 2016

\section{JEL classification:}

C70

D71

Keywords:

Feasible elimination procedure

Choosing $k$ from $m$

Axiomatization

Computation

\begin{abstract}
A B S T R A C T
We show that feasible elimination procedures (Peleg, 1978) can be used to select $k$ from $m$ alternatives. An important advantage of this method is the core property: no coalition can guarantee an outcome that is preferred by all its members. We also show that the problem of determining whether a specific $k$-tuple can result from a feasible elimination procedure is computationally equivalent to the problem of finding a maximal matching in a bipartite graph.
\end{abstract}

(C) 2016 Elsevier Inc. All rights reserved.

\section{Introduction}

This paper focuses on the question of how to choose a set of $k$ alternatives from a set of $m>k$ alternatives, by aggregating the preferences of voters over alternatives in a way that cannot be manipulated in the following sense: no coalition of voters, by possibly misrepresenting their preferences, can guarantee an outcome that all members of the coalition prefer to the outcome obtained by sincere, truthful voting. For instance, the set of $k$ alternatives is a department board, which has to be composed from a set of available candidates, and we are looking for a voting method such that no coalition of voters, by not voting sincerely, can guarantee a board that all members of the coalition prefer. In order to achieve this, we will use an extension of a method that was originally proposed by Peleg (1978) as a reaction to the Gibbard-Satterthwaite Theorem.

The Gibbard-Satterthwaite Theorem (Gibbard, 1973, and Satterthwaite, 1975) states that every non-dictatorial social choice function whose range contains at least three alternatives, is manipulable: there exists a profile of preferences and a voter who, by deviating and reporting a preference different from his true one, can obtain an outcome which he prefers, according to his true preference, to the sincere outcome. From a game-theoretic point of view, a social choice function combined with a (true) preference profile is a game in strategic form, the strategy set of each player being the set of preferences over the alternatives; outcomes of the game are evaluated according to the true preferences. Peleg (1978) showed that there are reasonable (anonymous, Maskin monotonic) social choice functions such that the resulting game has a strong Nash equilibrium resulting in the sincere outcome whatever the true preferences. Social choice functions with this

\footnotetext{
Financial support from GSBE, Maastricht University, is gratefully acknowledged. We thank an associate editor and two reviewers for helpful comments.

* Corresponding author.

E-mail addresses: pelegba@math.huji.ac.il (B. Peleg), h.peters@maastrichtuniversity.nl (H. Peters).
} 
property are called exactly and strongly consistent (ESC). In particular, for an ESC social choice function the sincere outcome is in the core of the relevant voting game: this means that there is no coalition of voters that has a strategy profile which guarantees an alternative that all members of the coalition prefer over the sincere outcome. Of course, the sincere outcome may not be the unique outcome of a strong Nash equilibrium. ${ }^{1}$

The central tool, introduced in Peleg (1978), to obtain these social choice functions is the concept of a feasible elimination procedure. In such a procedure, applied to a profile of preferences, alternatives are eliminated one by one, until a final alternative remains: this is called a maximal alternative. In this paper we use this procedure to select $k>1$ alternatives, simply by taking the last $k$ instead of only the last alternative. We show that for at least two extensions of voters' preferences over the alternatives to (ordered) $k$-tuples of alternatives, this method has the core property: no coalition can guarantee an outcome ( $k$-tuple) that is preferred by all its members. Formally, this core is defined as the core of the effectivity function induced by this method. We show, by an example, that some well-known existing methods (single transferable vote, plurality, plurality with run-off) violate the core property.

We also show that the problem of determining whether a specific $k$-tuple can result from a feasible elimination procedure is computationally equivalent to the problem of finding a maximal matching in a bipartite graph. The latter problem can be solved in polynomial time (Hopcroft and Karp, 1973).

Section 2 introduces feasible elimination procedures and Section 3 shows that these procedures provide a method to choose $k$ alternatives from a set of $m$ alternatives such that no coalition of voters can guarantee a better result by manipulation. We also show that this method is Maskin monotonic. Section 4 considers the computational aspect, and Section 5 concludes.

Notations. The following basic notations are used throughout. For a set $D,|D|$ denotes the cardinality of $D, P(D)$ the power set, i.e., the set of all subsets of $D$, and $P_{0}(D)$ the set of all nonempty subsets of $D$.

\section{Preliminaries}

Let $A$ be a set of $m$ alternatives, $m \geq 2$, and let $N=\{1, \ldots, n\}, n \geq 2$, be a set of voters. Denote by $L$ the set of all linear orderings, i.e., complete, antisymmetric and transitive binary relations, of $A$. An element of $L$ is also called a preference, and an element of $L^{N}$ a preference profile. If $x R^{i} y$ for some $x, y \in A$ and $i \in N$, where $R^{i} \in L$, then this is interpreted as voter $i$ strictly preferring $x$ over $y$.

A social choice correspondence (SCC) is a function $H: L^{N} \rightarrow P_{0}(A)$. An SCC $H$ is anonymous if for all $R^{N} \in L^{N}$ and for all permutations $\pi$ of $N, H\left(R^{1}, \ldots, R^{n}\right)=H\left(R^{\pi(1)}, \ldots, R^{\pi(n)}\right)$. It is Paretian if for all $x, y \in A$ and $R^{N} \in L^{N}$, if $x \neq y$ and $y R^{i} x$ for all $i \in N$, then $x \notin H\left(R^{N}\right)$. It is Maskin monotonic (Maskin, 1999) if it satisfies the following. Let $R^{N}, Q^{N} \in L^{N}$ and let $x \in H\left(Q^{N}\right)$. If $x Q^{i} y$ implies $x R^{i} y$ for all $y \in A$ and $i \in N$, then $x \in H\left(R^{N}\right)$.

In this paper we are especially interested in the SCC derived from feasible elimination procedures, introduced by Peleg (1978).

Definition 2.1. Assume that $n+1 \geq m$ and let $\beta: A \rightarrow\{1,2 \ldots\}$ satisfy $\sum_{x \in A} \beta(x)=n+1$. Let $R^{N} \in L^{N}$. A feasible elimination procedure (f.e.p.) for $R^{N}$ is a sequence $\left(x_{1}, C_{1} ; \ldots ; x_{m-1}, C_{m-1} ; x_{m}\right)$ such that

1) $A=\left\{x_{1}, \ldots, x_{m}\right\}$

2) $C_{1}, \ldots, C_{m-1}$ are pairwise disjoint subsets of $N$ and $\left|C_{j}\right|=\beta\left(x_{j}\right)$ for $j=1, \ldots, m-1$,

3) For all $j=1, \ldots, m-1, x_{k} R^{i} x_{j}$ for all $k=j+1, \ldots, m$ and $i \in C_{j}$.

Thus, in a feasible elimination procedure, we can consecutively eliminate bottom alternatives $x_{1}, x_{2}, \ldots, x_{m-1}$ of the preference profile; when we eliminate an alternative $x_{j}$ we also eliminate the preferences of $\beta\left(x_{j}\right)$ voters who have $x_{j}$ at bottom in the current profile. From the assumptions in the definition it easily follows that there exists always at least one f.e.p. Henceforth in this paper we assume $n+1 \geq m$. An alternative $y$ is $R^{N}$-maximal if there exists an f.e.p. $\left(x_{1}, C_{1} ; \ldots ; x_{m-1}, C_{m-1} ; y\right)$. We denote

$$
M\left(R^{N}\right)=\left\{x \in A: x \text { is } R^{N} \text {-maximal }\right\} .
$$

It is not difficult to see that $M$ is an anonymous and Paretian social choice correspondence. ${ }^{2}$ Also, $M$ is Maskin monotonic. This follows from Lemma 3.7 below.

\footnotetext{
1 Peleg (1978) has been followed by several investigations of the set of exactly and strongly consistent social choice functions: Dutta and Pattanaik (1978), Polishchuk (1978), Ishikawa and Nakamura (1980), Oren (1981), Kim and Roush (1981), Holzman (1986), and Peleg and Peters (2006). Also the books of Peleg (1984), Abdou and Keiding (1991), Danilov and Sotskov (2002), and Peleg and Peters (2010) contain lengthy analytic discussions of consistent voting systems.

2 Clearly, $M$ depends on $\beta$, but this is suppressed from notation if no confusion is likely.
} 
Remark 2.2. As mentioned in the Introduction, the interest of feasible elimination procedures and the SCC $M$ lies in the fact that, for every social choice function $F$ (i.e., single-valued SCC) that is a selection from $M$, the game in which each voter reports a preference and the outcome is determined by $F$, has a strong Nash equilibrium resulting in the sincere outcome, i.e., the alternative resulting if the players would report truthfully. This provides a way to obtain the sincere outcome in spite of the Gibbard-Satterthwaite result that any reasonable social choice function is manipulable. In fact, it can be shown that under anonymity and a very reasonable additional condition, namely that no single voter can veto an alternative, selecting from maximal alternatives is the only way to achieve this (see Peleg and Peters, 2010).

\section{Choosing $k$ from $m$}

In this section we show how the concept of a feasible elimination procedure can be used to select not just one alternative, but an ordered $k$-tuple of alternatives, where $1 \leq k \leq m-1$. We show, in particular, that the resulting method has the 'core property': there is no subset of voters that can vote strategically in order to guarantee a better outcome for all its members. This is in contrast to some well-known existing methods that can be used for choosing $k$ from $m-$ see Example 3.6.

In order to formalize this we use the concept of an effectivity function, defined here for some general finite set of alternatives $Z$.

Definition 3.1. A function $E: P(N) \rightarrow P\left(P_{0}(Z)\right)$ is an effectivity function (EF) if: 1$) E(N)=P_{0}(Z)$, 2) $Z \in E(S)$ for every $S \in P_{0}(N)$, and 3) $E(\emptyset)=\emptyset$.

If $B \in E(S)$ for an effectivity function $E$, then we say that coalition $S$ is effective for the set of alternatives $B$. The interpretation is that $S$ can 'guarantee' that the 'final' alternative is in $B$.

Let $k \in\{1, \ldots, m-1\}$ and denote by $\bar{A}^{k}$ the set

$$
\left\{\left(x_{1}, \ldots, x_{k}\right) \in A^{k}:\left|\left\{x_{1}, \ldots, x_{k}\right\}\right|=k\right\} .
$$

As before, assume that $n+1 \geq m$ and let $\beta: A \rightarrow N$ satisfy $\sum_{x \in A} \beta(x)=n+1$. For these weights, we define $M^{k}: L^{N} \rightarrow$ $P_{0}\left(\bar{A}^{k}\right)$ by: $\left(y_{1}, \ldots, y_{k}\right) \in M^{k}\left(R^{N}\right)$ if there is an f.e.p. $\left(x_{1}, C_{1} ; \ldots ; x_{m-1}, C_{m-1} ; x_{m}\right)$ such that $\left(y_{1}, \ldots, y_{k}\right)=\left(x_{m-k+1}, \ldots, x_{m}\right)$ In words, $M^{k}$ assigns to a preference profile all tuples of $k$ last alternatives in any f.e.p. for that profile. Clearly, $M^{1}=M$. Define the function $E^{M^{k}}: P(N) \rightarrow P\left(P_{0}\left(\bar{A}^{k}\right)\right)$ by $E^{M^{k}}(\emptyset)=\emptyset$ and, for $S \neq \emptyset$ and $B^{k} \subseteq \bar{A}^{k}$ :

$$
B^{k} \in E^{M^{k}}(S): \Leftrightarrow \exists R^{S} \in L^{S}\left[\forall R^{N \backslash S} \in L^{N \backslash S}: M^{k}\left(R^{S}, R^{N \backslash S}\right) \subseteq B^{k}\right] .
$$

Since, in particular, $\left\{\left(x_{1}, \ldots, x_{k}\right)\right\} \in E^{M^{k}}(N)$ for each $\left(x_{1}, \ldots, x_{k}\right) \in \bar{A}^{k}$ (namely, for each $i \in N$ let $R^{i}$ satisfy $x_{k} R^{i} x_{k-1} \ldots$ $\left.x_{2} R^{i} x_{1} R^{i} \ldots\right)$, we have that $E^{M^{k}}$ is an effectivity function, with $Z=\bar{A}^{k}$ in Definition 3.1 .

In this paper we consider two kinds of preferences of the voters for $k$-tuples. Both kinds of preferences extend the original preferences in $L$ to linear orderings on $\bar{A}^{k}$. In the first extension we respect the order of a $k$-tuple and lexicographically compare alternatives, starting from the rightmost one. Formally we have:

(P1) Let $R \in L$. Then for $\bar{x}=\left(x_{1}, \ldots, x_{k}\right) \in \bar{A}^{k}$ and $\bar{y}=\left(y_{1}, \ldots, y_{k}\right) \in \bar{A}^{k}$ we define $\bar{x} \bar{R} \bar{y}$ if $x_{\ell} R y_{\ell}$, where $\ell=\max \{j \in$ $\left.\{1, \ldots, k\}: x_{j} \neq y_{j}\right\}$.

In the second extension we ignore the order of a $k$-tuple and lexicographically compare alternatives, starting from the worst one. Formally we have:

(P2) Let $R \in L$. For $\bar{x}=\left(x_{1}, \ldots, x_{k}\right) \in \bar{A}^{k}$ and $\bar{y}=\left(y_{1}, \ldots, y_{k}\right) \in \bar{A}^{k}$ reorder the alternatives so that $x_{i_{1}} R \ldots R x_{i_{k}}$ and $y_{j_{1}} R \ldots R y_{j_{k}}$. Then we define $\bar{x} \bar{R} \bar{y}$ if $x_{i_{\ell}} R y_{j_{\ell}}$, where $\ell=\max \left\{h \in\{1, \ldots, k\}: x_{i_{h}} \neq y_{j_{h}}\right\}$.

Preference extension P1 could apply, for instance, if a president and a vice-president of a society have to be chosen $(k=2)$. Then we first compare the candidates for president and, in case these are equal, we compare the candidates for vice-president. Extension P2 could apply when the members of a board to be chosen are on equal foot. Then we first compare the worst candidates. If these are the same, then we compare second worst candidates, etc.

In what follows we concentrate on these two preference extensions. For another extension and discussion, see Section 5. Before we can formulate the main results of this section we need another definition.

Definition 3.2. Let $E: P(N) \rightarrow P\left(P_{0}(Z)\right)$ be an EF, let $R^{N}$ be a profile of linear orderings over $Z$, let $S \in P_{0}(N)$, let $B \in E(S)$, and let $z \in Z \backslash B$. We say that $z$ is dominated by $B$ via $S$ at $R^{N}$ if $z^{\prime} R^{i} z$ for all $z^{\prime} \in B$ and $i \in S$; $z$ is dominated at $R^{N}$ if there exist $B$ and $S$ as above such that $B$ dominates $z$ via $S$ at $R^{N}$. The core of $E$ at $R^{N}, C\left(E, R^{N}\right)$, is the set of all alternatives that are not dominated at $R^{N}$. 
We will now show that $M^{k}$ selects only core alternatives under both preference extensions (P1) and (P2), starting with the latter.

Theorem 3.3. Let $R^{N} \in L^{N}$, and let $\bar{R}^{N}$ be the profile of extended preferences according to (P2). Then $M^{k}\left(R^{N}\right) \subseteq C\left(E^{M^{k}}, \bar{R}^{N}\right)$.

Proof. Consider an f.e.p. $f^{*}=\left(x_{1}, C_{1} ; \ldots ; x_{m-1}, C_{m-1} ; x_{m}\right)$ for $R^{N}$, resulting in $\left(x_{m-k+1}, \ldots, x_{m}\right) \in M^{k}\left(R^{N}\right)$. Suppose there is an $S \subseteq N$ and a set $B^{k} \subseteq \bar{A}^{k}$ such that $B^{k} \in E^{M^{k}}(S),\left(x_{m-k+1}, \ldots, x_{m}\right) \notin B^{k}$, and $\bar{y} \bar{R}^{i}\left(x_{m-k+1}, \ldots, x_{m}\right)$ for all $i \in S$ and $\bar{y} \in B^{k}$. We derive a contradiction, which completes the proof of the theorem.

Let $K=\left\{x_{m-k+1}, \ldots, x_{m}\right\}$ and let

$$
B=\left\{x \in A: x \in\left\{y_{1}, \ldots, y_{k}\right\} \text { for some }\left(y_{1}, \ldots, y_{k}\right) \in B^{k}\right\} .
$$

Note that $B \backslash K \neq \emptyset$, otherwise we would have $B=K$, which is not possible in view of the preferences of the voters in $S$. Consider any $x_{j} \in B \backslash K$, and $\bar{y}=\left(y_{1}, \ldots, y_{k}\right) \in B^{k}$ with $y_{\ell}=x_{j}$ for some $\ell \in\{1, \ldots, k\}$. Since $\bar{y} \bar{R}^{i}\left(x_{m-k+1}, \ldots, x_{m}\right)$ for all $i \in S$, by (P2) there is for each $i \in S$ an $x_{h} \in K$ with $x_{j} R^{i} x_{h}$. Since $x_{j}$ is eliminated according to $f^{*}$ prior to the alternatives of $K$, this implies that $C_{j} \cap S=\emptyset$. Hence, the alternatives of $B \backslash K$ are eliminated according to $f^{*}$ via only voters in $N \backslash S$. Therefore we have $|N \backslash S| \geq \beta(B \backslash K)$.

Let $Q^{S} \in L^{S}$ be a profile resulting in $B^{k}$, i.e., $M^{k}\left(Q^{S}, V^{N \backslash S}\right) \subseteq B^{k}$ for all $V^{N \backslash S} \in L^{N \backslash S}$. Consider, in particular, a profile $V^{N \backslash S} \in L^{N \backslash S}$ such that each $x_{j} \in B \backslash K$ is a bottom alternative for at least $\beta\left(x_{j}\right)$ voters in $N \backslash S$ (this is possible since $|N \backslash S| \geq$ $\beta(B \backslash K))$. Then, for this specific profile $\left(Q^{S}, V^{N \backslash S}\right)$, there is an f.e.p. in which all alternatives of $B \backslash K$ are eliminated first. This implies that the resulting $k$-tuple, say $\bar{y}$, of $B^{k}$ can only contain alternatives of $K$, contradicting $\bar{y} \bar{R}^{i}\left(x_{m-k+1}, \ldots, x_{m}\right)$ for all $i \in S$.

Theorem 3.4. Let $R^{N} \in L^{N}$, and let $\bar{R}^{N}$ be the profile of extended preferences according to (P1). Then $M^{k}\left(R^{N}\right) \subseteq C\left(E^{M^{k}}, \bar{R}^{N}\right)$.

Proof. Let $f^{*}, S, B^{k}$, and $Q^{S}$ be as in the proof of Theorem 3.3. The proof proceeds in several steps.

Step 1 Let

$$
\begin{aligned}
B(m)= & \left\{x_{j} \in A: j \in\{1, \ldots, m-1\}\right. \text { and } \\
& \left.x_{j}=y_{k} \text { for some }\left(y_{1}, \ldots, y_{k}\right) \in B^{k}\right\} .
\end{aligned}
$$

By (P1), we have $x_{j} R^{i} x_{m}$ for all $x_{j} \in B(m)$ and $i \in S$. Hence, $C_{j} \subseteq N \backslash S$ for all $j$ with $x_{j} \in B(m)$, so that $|N \backslash S| \geq \beta(B(m))$. Consider a profile $V^{N \backslash S}(m) \in L^{N \backslash S}$ where all $x_{j} \in B(m)$ are ranked at bottom positions for at least $\beta\left(x_{j}\right)$ voters in $N \backslash S$. Let $B^{k}(m)$ be the subset of $B^{k}$, consisting of all $k$-tuples which are obtained by f.e.p.'s for the profile $\left(Q^{S}, V^{N \backslash S}(m)\right)$ such that the alternatives of $B(m)$ are eliminated first, with $x_{j}$ before $x_{\ell}$ whenever $x_{j}, x_{\ell} \in B(m)$ and $j<\ell$; clearly, all these f.e.p.'s result in $x_{m}$, so that $y_{k}=x_{m}$ for all $\left(y_{1}, \ldots, y_{k}\right) \in B^{k}(m)$.

Step 2 Let

$$
\begin{aligned}
B(m-1)= & \left\{x_{j}: j \in\{1, \ldots, m-2\}\right. \text { and } \\
& \left.x_{j}=y_{k-1} \text { for some }\left(y_{1}, \ldots, y_{k-1}, x_{m}\right) \in B^{k}(m)\right\} .
\end{aligned}
$$

By (P1), we have $x_{j} R^{i} x_{m-1}$ for all $x_{j} \in B(m-1)$ and $i \in S$. Hence, $C_{j} \subseteq N \backslash S$ for all $j$ with $x_{j} \in B(m-1)$, so that $|N \backslash S| \geq$ $\beta(B(m) \cup B(m-1))$. Consider a profile $V^{N \backslash S}(m-1) \in L^{N \backslash S}$ where all $x_{j} \in B(m) \cup B(m-1)$ are ranked at bottom positions for at least $\beta\left(x_{j}\right)$ voters in $N \backslash S$. Let $B^{k}(m-1)$ be the subset of $B^{k}(m)$, consisting of all $k$-tuples which are obtained by f.e.p.'s for the profile $\left(Q^{S}, V^{N \backslash S}(m-1)\right)$ such that the alternatives of $B(m) \cup B(m-1)$ are eliminated first, with $x_{j}$ before $x_{\ell}$ whenever $x_{j}, x_{\ell} \in B(m) \cup B(m-1)$ and $j<\ell$; clearly, all these f.e.p.'s have $\left(x_{m-1}, x_{m}\right)$ as last pair, so that $y_{k-1}=x_{m-1}$ and $y_{k}=x_{m}$ for all $\left(y_{1}, \ldots, y_{k}\right) \in B^{k}(m-1)$.

$\underline{\text { Step k Let }}$

$$
\begin{aligned}
B(m-k+1)= & \left\{x_{j}: j \in\{1, \ldots, m-k\}\right. \text { and } \\
& \left.x_{j}=y_{1} \text { for some }\left(y_{1}, x_{m-k+2}, \ldots, x_{m}\right) \in B^{k}(m-k+2)\right\} .
\end{aligned}
$$

By (P1), we have $x_{j} R^{i} x_{m-k+1}$ for all $x_{j} \in B(m-k+1)$ and $i \in S$. Hence, $C_{j} \subseteq N \backslash S$ for all $j$ with $x_{j} \in B(m-k+1)$, so that $|N \backslash S| \geq \beta(B(m) \cup \ldots \cup B(m-k+1))$. Consider a profile $V^{N \backslash S}(m-k+1) \in L^{N \backslash S}$ where all $x_{j} \in B(m) \cup \ldots \cup B(m-k+1)$ are ranked at bottom positions for at least $\beta\left(x_{j}\right)$ voters in $N \backslash S$. Let $B^{k}(m-k+1)$ be the subset of $B^{k}(m-k+2)$, consisting of all $k$-tuples which are obtained by f.e.p.'s for the profile $\left(Q^{S}, V^{N \backslash S}(m-k+1)\right)$ such that the alternatives of $B(m) \cup \ldots \cup$ 
$B(m-k+1)$ are eliminated first, with $x_{j}$ before $x_{\ell}$ whenever $x_{j}, x_{\ell} \in B(m) \cup \ldots \cup B(m-k+1)$ and $j<\ell$; clearly, all these f.e.p.'s have $\left(x_{m-k+1}, \ldots, x_{m}\right)$ as last $k$-tuple. This, however, implies that $\left(x_{m-k+1}, \ldots, x_{m}\right) \in B^{k}$, which is a contradiction.

The following example shows that the set inclusions in Theorems 3.3 and 3.4 can be strict if $k>1$. (For $k=1$ we do have $M\left(R^{N}\right)=C\left(E^{M}, R^{N}\right)$ for all $R^{N} \in L^{N}$ by Lemma 9.3.2 and Theorem 9.3.6 in Peleg and Peters, 2010.)

Example 3.5. Let $A=\{x, y, z\}, N=\{1, \ldots, 4\}, \beta(x)=1$, and $\beta(y)=\beta(z)=2$. Consider the profile $R^{N}$ given in the following table:

\begin{tabular}{llll}
\hline$R^{1}$ & $R^{2}$ & $R^{3}$ & $R^{4}$ \\
\hline$y$ & $y$ & $x$ & $x$ \\
$z$ & $z$ & $z$ & $y$ \\
$x$ & $x$ & $y$ & $z$ \\
\hline
\end{tabular}

Then $M^{2}\left(R^{N}\right)=\{(z, y)\}$. We claim that the pair $(x, y)$ is in $C\left(E^{M^{2}}, \bar{R}^{N}\right)$ according to preference assumption (P2). First, $(x, y)$ is top-ranked for voter 4 . Voter 3 only prefers $(x, z)$ or $(z, x)$ to $(x, y)$ but this is not the case for voters 1 and 2 . However, voter 3 alone is not effective for $\{(x, z),(z, x)\}$. Finally, voters 1 and 2 only prefer $(y, z)$ and $(z, y)$ to $(x, y)$, but $\{1,2\}$ is not effective for $\{(y, z),(z, y)\}$. We conclude that $(x, y)$ is in $C\left(E^{M^{2}}, \bar{R}^{N}\right)$ under assumption (P2).

Now consider preference assumption (P1). We claim that $(x, y)$ is still in $C\left(E^{M^{2}}, \bar{R}^{N}\right)$. Voter 4 finds $(z, x)$ and $(y, x)$ better than $(x, y)$; also voter 3 finds $(z, x)$ and $(y, x)$ better than $(x, y)$, but voters 1 and 2 prefer $(x, y)$ over $(z, x)$ and $(y, x)$, and $\{3,4\}$ is not effective for $\{(z, x),(y, x)\}$ : e.g., $\{1,2\}$ can put $x$ at bottom so that $x$ can be eliminated first. Voter 3 prefers every alternative in the set $\{(y, x),(z, x),(y, z),(x, z)\}$ to $(x, y)$, but 1 and 2 prefer only $(z, y)$ over $(x, y)$, and $\{3\}$ is not effective for $\{(y, x),(z, x),(y, z),(x, z)\}:\{1,2,4\}$ can put $x$ and $z$ at bottom so that $y$ ends up last. Finally, $\{1,2\}$ is not effective for $\{(z, y)\}$ : e.g., $\{3,4\}$ can put $z$ at bottom so that $z$ can be eliminated first. We conclude that $(x, y)$ is in $C\left(E^{M^{2}}, \bar{R}^{N}\right)$ also under assumption (P1).

The fact that $M^{k}$ satisfies the core property as in Theorems 3.3 and 3.4 means that there is never a coalition of voters which can guarantee an outcome ( $k$-tuple) that is better for all its members in case $M^{k}$ is used, i.e., if a feasible elimination procedure is used. This fact is certainly not shared by well-known existing methods. The following example illustrates this.

Example 3.6. Let $A=\{a, b, c, d\}, N=\{1,2,3\}$, and $\beta(x)=1$ for all $x \in A$. Consider the profile $R^{N}$ given in the following table:

\begin{tabular}{lll}
\hline$R^{1}$ & $R^{2}$ & $R^{3}$ \\
\hline$a$ & $b$ & $c$ \\
$b$ & $c$ & $a$ \\
$c$ & $a$ & $b$ \\
$d$ & $d$ & $d$
\end{tabular}

Suppose we have to select two candidates. In this case, $M^{2}$ is quite inconclusive: $M^{2}\left(R^{N}\right)=\left\{(x, y) \in \bar{A}^{2}: x \neq d, y \neq d\right\}$. Still by Theorems 3.3 and 3.4, no coalition of voters can improve on any of the pairs in $M^{2}\left(R^{N}\right)$. Now consider the method of single transferable vote (STV - see for instance Brams and Fishburn, 2002). According to STV, first $d$ is eliminated, but thereafter either $a$ or $b$ or $c$ is eliminated. For instance, if $a$ is eliminated, then next $b$ is chosen and finally $c$, so that in our notation the pair $(c, b)$ results. This way, we obtain $\operatorname{STV}\left(R^{N}\right)=\{(c, b),(b, a),(a, c)\}$. Now take, for instance, the pair $(b, a)$. Both voters 2 and 3 prefer the pair $(b, c)$ over $(b, a)$ - under both (P1) and (P2) - and, moreover, under STV the coalition $\{2,3\}$ is effective for $\{(b, c)\}$ by the strategy profile

\begin{tabular}{ll}
\hline$Q^{2}$ & $Q^{3}$ \\
\hline$c$ & $c$ \\
$b$ & $b$ \\
$a$ & $a$ \\
$d$ & $d$ \\
\hline
\end{tabular}

In fact, under STV the core for the profile in this example (which is a slightly modified version of the Condorcet paradox) is empty. It is easy to see that the same example applies to methods like plurality voting or run-off elections, assuming that in case of ties we can choose arbitrarily between tied alternatives, as in STV or feasible elimination procedures. 
We conclude this section with showing that the SCC $M^{k}$ is Maskin monotonic. This result will be used in Section 4, but is also of independent interest. A function $H: L^{N} \rightarrow P_{0}\left(\bar{A}^{k}\right)$ is Maskin monotonic if it satisfies the following. Let $R^{N}, Q^{N} \in L^{N}$ and let $\left(x_{1}, \ldots, x_{k}\right) \in H\left(Q^{N}\right)$. If $x_{j} Q^{i} y$ implies $x_{j} R^{i} y$ for all $j \in\{1, \ldots, k\}, y \in A$ and $i \in N$, then $\left(x_{1}, \ldots, x_{k}\right) \in H\left(R^{N}\right)$. For $k=1$, this definition coincides with the definition of Maskin monotonicity in Section 2 . $^{3}$

\section{Lemma 3.7. $M^{k}$ is Maskin monotonic.}

Proof. Let $Q^{N}$ and $R^{N}$ as in the definition of Maskin monotonicity. Without loss of generality we assume that there is a voter $v$ such that $Q^{N \backslash\{v\}}=R^{N \backslash\{v\}}$. Let $f^{*}=\left(x_{1}, C_{1} ; \ldots ; x_{m-1}, C_{m-1} ; x_{m}\right)$ be an f.e.p. for $Q^{N}$. If $v \notin C_{1} \cup \ldots \cup C_{m-k}$ then it is easy to see that $f^{*}$ is still an f.e.p. for $R^{N}$, so that $\left(x_{m-k+1}, \ldots, x_{m}\right) \in M^{k}\left(R^{N}\right)$. Now assume $v \in C_{1} \cup \ldots \cup C_{m-k}$. If $v \in C_{j}$ with $j>1$, then we may eliminate $x_{1}, \ldots, x_{j-1}$ and all voters in $C_{1} \cup \ldots \cup C_{j-1}$ first, and next continue the argument with the remaining profile, where now all voters in $C_{j}$ have $x_{j}$ bottom ranked according to $Q$. So, without loss of generality, let $v \in C_{1}$.

The rest of the proof is based on a three step algorithm. Step 2 .

Step 1 If the bottom alternative of $R^{v}$ is equal to $x_{1}$, then $f^{*}$ is still an f.e.p. for $R^{N}$ and we are done. Otherwise, go to

Step 2 Let the bottom alternative of $R^{v}$ be $x_{\ell} \neq x_{1}$, so $\ell \in\{2, \ldots, m-k\}$. If all voters in $C_{\ell}$ have $x_{\ell}$ as bottom alternative in $\overline{R^{N}}$, then we can first eliminate $x_{\ell}$ via $C_{\ell}$ and go back to Step 1 for the reduced profile. Otherwise, go to Step 3.

Step 3 Take $\hat{v} \in C_{\ell}$ with $x_{\ell}$ not as bottom alternative and note that the bottom alternative of $R^{\hat{v}}=Q^{\hat{v}}$ is some $x_{j}$ with $j<\ell$ (since $x_{j}$ must be eliminated before $x_{\ell}$ in $f^{*}$ ). Then modify $C_{\ell}$ to $\hat{C}_{\ell}=\left(C_{\ell} \cup\{v\}\right) \backslash\{\hat{v}\}$ and modify $C_{1}$ to $\hat{C}_{1}=\left(C_{1} \cup\{\hat{v}\}\right) \backslash\{v\}$. (In words, we switch $v$ and $\hat{v}$.) Go back to Step 1 .

Repeat this procedure until the final substitute of $v$ in the modified $C_{1}$ has $x_{1}$ at bottom. Then we can apply an f.e.p. resulting in $\left(x_{m-k+1}, \ldots, x_{m}\right)$, so that $\left(x_{m-k+1}, \ldots, x_{m}\right) \in M^{k}\left(R^{N}\right)$.

For $k=1$, Maskin monotonicity was already proved in Peleg (1984), Lemma 5.3.5.

\section{A method for computing $M^{k}$ in polynomial time}

In this section we show that the SCC $M^{k}$ can be computed in polynomial time. The argument will be based on the following lemma, which, in turn, follows from Maskin monotonicity of $M^{k}$. Throughout, $M^{k}$ is assumed to be defined with respect to weights $\beta(x), x \in A$.

Lemma 4.1. Let $R^{N} \in L^{N}$. Then $\left(x_{m-k+1}, \ldots, x_{m}\right) \in M^{k}\left(R^{N}\right)$ if and only if there exist pairwise disjoint subsets $S(y), y \in A \backslash\left\{x_{m}\right\}$, of $N$ such that

(1) $x_{j} R^{i} x_{\ell}$ for all $\ell=m-k+1, \ldots, m-1, j>\ell$, and $i \in S\left(x_{\ell}\right)$,

(2) $x_{j} R^{i} y$ for all $y \in A \backslash\left\{x_{m-k+1}, \ldots, x_{m}\right\}, j=m-k+1, \ldots, m$, and $i \in S(y)$,

(3) $|S(y)|=\beta(y)$ for all $y \in A \backslash\left\{x_{m}\right\}$.

Proof. The only-if direction follows immediately from the definition of an f.e.p. For the if-direction, let $\left(x_{m-k+1}, \ldots, x_{m}\right) \in \bar{A}^{k}$ and assume that there exist subsets $S(y)$ of $N$, satisfying (1), (2), and (3). List the alternatives of $A \backslash\left\{x_{m-k+1}, \ldots, x_{m}\right\}$ as $\left\{x_{1}, \ldots, x_{m-k}\right\}$. Consider the following profile $Q^{N} \in L^{N}$. For $j=1, \ldots, m-k$ and $i \in S\left(x_{j}\right)$ shift $x_{j}$ to the bottom of $R^{i}$, leaving everything else intact. For all other voters $i$, let $Q^{i}=R^{i}$. Then $\left(x_{1}, S\left(x_{1}\right) ; \ldots ; x_{m-1}, S\left(x_{m-1}\right) ; x_{m}\right)$ is an f.e.p. for $Q^{N}$, so that $\left(x_{m-k+1}, \ldots, x_{m}\right) \in M^{k}\left(Q^{N}\right)$. By Maskin monotonicity of $M^{k}$ (see Lemma 3.7), $\left(x_{m-k+1}, \ldots, x_{m}\right) \in M^{k}\left(R^{N}\right)$.

Lemma 4.1 can be used to determine if an alternative $\left(x_{m-k+1}, \ldots, x_{m}\right)$ of $\bar{A}^{k}$, is in $M^{k}\left(R^{N}\right)$. We first collect a few graph-theoretical concepts in the following definition.

Definition 4.2. A bipartite graph is a triple $(X, Y, W)$, where $X$ and $Y$ are finite disjoint sets and $W \subseteq X \times Y$. Here, the elements of $X$ and $Y$ are called vertices and the elements of $W$ edges. A matching is a set $V \subseteq W$ such that for all $(x, y),\left(x, y^{\prime}\right),\left(x^{\prime}, y\right) \in V$ we have $y=y^{\prime}$ and $x=x^{\prime}$. A matching $V$ is maximal if there is no matching $V^{\prime}$ that has more edges, i.e., there is no matching $V^{\prime}$ with $\left|V^{\prime}\right|>|V|$.

For $\left(x_{m-k+1}, \ldots, x_{m}\right) \in \bar{A}^{k}$ we define a bipartite graph $(X, Y, W)$ as follows. Let $X=N$ and $Y=\left\{x_{\ell}^{1}, \ldots, x_{\ell}^{\beta\left(x_{\ell}\right)}: \ell=\right.$ $1, \ldots, m-1\}$, where the alternatives in $A \backslash\left\{x_{m-k+1}, \ldots, x_{m}\right\}$ are listed as $\left\{x_{1}, \ldots, x_{m-k}\right\}$. In other words, the vertices in $X$

\footnotetext{
3 Also note that this extended definition of Maskin monotonicity would be implied by Maskin monotonicity in terms of extended preferences according to (P1) or (P2) or any other sensible preference extension.
} 
correspond to the voters and the vertices in $Y$ to the alternatives except $x_{m}$, where for each $x_{\ell}$ we have $\beta\left(x_{\ell}\right)$ vertices. We define $W$ by

$$
W=\left\{\left(i, x_{\ell}^{p}\right) \in X \times Y: x_{j} R^{i} x_{\ell} \text { for all } j \in\{m-k+1, \ldots, m\} \text { with } j>\ell\right\} .
$$

Thus, for every vertex corresponding to an alternative $x_{\ell} \in A \backslash\left\{x_{m-k+1}, \ldots, x_{m}\right\}$, we let there be an edge between this vertex and the vertex corresponding to a voter $i$ if and only if $x_{j} R^{i} x_{\ell}$ for every $j=m-k+1, \ldots, m$. For every vertex corresponding to an alternative $x_{\ell}$ for some $\ell \in\{m-k+1, \ldots, m-1\}$, we let there be an edge between this vertex and the vertex corresponding to a voter $i$ if and only if $x_{j} R^{i} x_{\ell}$ for all $j=\ell+1, \ldots, m$.

We now have the following lemma.

Lemma 4.3. Let $R^{N} \in L^{N}$. Then $\left(x_{m-k+1}, \ldots, x_{m}\right) \in M^{k}\left(R^{N}\right)$ if and only if the associated graph $(X, Y, W)$ has a matching $V$ such that for every $x_{\ell}^{p} \in Y$ there is an $i \in X$ with $\left(i, x_{\ell}^{p}\right) \in V$.

Proof. First, suppose that $\left(x_{m-k+1}, \ldots, x_{m}\right) \in M^{k}\left(R^{N}\right)$. Let the sets $S\left(x_{\ell}\right), \ell=1, \ldots, m-1$ be as in Lemma 4.1 . Then for each vertex $x_{\ell}^{p} \in Y$ there is an $i_{\ell}^{p} \in S\left(x_{\ell}\right) \subseteq X$ such that $\left(i_{\ell}^{p}, x_{\ell}^{p}\right) \in W$ and such that all $i_{\ell}^{p}$ are different. Thus, the set $\left\{\left(i_{\ell}^{p}, x_{\ell}^{p}\right): x_{\ell}^{p} \in Y\right\}$ is a matching in $(X, Y, W)$ satisfying the requirement for $V$ in the lemma.

For the converse, suppose that $V$ is a matching in $(X, Y, W)$ as in the lemma. For every $x_{\ell} \neq x_{m}$ let $S\left(x_{\ell}\right)=\{i \in X$ : $\left(i, x_{\ell}^{p}\right) \in V$ for some $\left.1 \leq p \leq \beta\left(x_{\ell}\right)\right\}$. Then it is easy to verify that these sets $S\left(x_{\ell}\right)$ satisfy the conditions in Lemma 4.1 . Hence, $\left(x_{m-k+1}, \ldots, x_{m}\right) \in M^{k}\left(R^{N}\right)$.

Note that a matching as in Lemma 4.3 must be maximal, since it contains an edge for every vertex in $Y$. Thus, by this lemma, in order to decide if $\left(x_{m-k+1}, \ldots, x_{m}\right) \in M^{k}\left(R^{N}\right)$ it is sufficient to find a maximal matching of the associated bipartite graph $(X, Y, W)$ : then $\left(x_{m-k+1}, \ldots, x_{m}\right) \in M^{k}\left(R^{N}\right)$ if and only if the found maximal matching uses all vertices of $Y$. The problem of finding a maximal matching is polynomial (see Hopcroft and Karp, 1973). Repeating the foregoing procedure $m(m-1) \cdots(m-k+1)$ times is still polynomial (in $m$ and $n){ }^{4}$

\section{Concluding remarks}

We conclude with a few brief discussions.

\subsection{Other preference extensions}

We have shown in this paper that the method for choosing $k$ out of $m$, based on feasible elimination procedures, has the core property for two intuitive preferences extensions (Theorems 3.3 and 3.4). Unfortunately, as the following example shows, this result does not extend to all reasonable preference extensions.

Example 5.1. Let $A=\{w, x, y, z\}, N=\{1, \ldots, 5\}, \beta(w)=\beta(x)=1$, and $\beta(y)=\beta(z)=2$. Consider the profile $R^{N}$ given in the following table:

\begin{tabular}{lllll}
\hline$R^{1}$ & $R^{2}$ & $R^{3}$ & $R^{4}$ & $R^{5}$ \\
\hline$y$ & $y$ & $z$ & $z$ & $x$ \\
$w$ & $w$ & $w$ & $w$ & $y$ \\
$x$ & $x$ & $x$ & $x$ & $z$ \\
$z$ & $z$ & $y$ & $y$ & $w$ \\
\hline
\end{tabular}

Then $(w, x) \in M^{2}\left(R^{N}\right)$ by the f.e.p. $(z,\{1,2\} ; y,\{3,4\} ; w,\{5\} ; x)$. We show that $(w, x) \neq C\left(E^{M^{2}}, \bar{R}^{N}\right)$ where, for each $i \in N$, $\bar{R}^{i}$ is the lexicographic preference extension obtained by first comparing best alternatives and then second best alternatives. Consider the following profile for the coalition $\{1, \ldots, 4\}$ :

\begin{tabular}{llll}
\hline$Q^{1}$ & $Q^{2}$ & $Q^{3}$ & $Q^{4}$ \\
\hline$y$ & $y$ & $y$ & $y$ \\
$z$ & $z$ & $z$ & $z$ \\
$x$ & $x$ & $x$ & $x$ \\
$w$ & $w$ & $w$ & $w$ \\
\hline
\end{tabular}

\footnotetext{
${ }^{4}$ We thank Ilan Nehama of the Center for the Study of Rationality, Jerusalem, for helpful comments on this subject.
} 
Then $M^{2}\left(Q^{\{1, \ldots, 4\}}, \widetilde{R}^{5}\right)=\{(z, y)\}$ for all $\widetilde{R}^{5} \in L$, which implies that $\{(z, y)\} \in E^{M^{2}}(\{1, \ldots, 4\})$. Since $(z, y) \bar{R}^{i}(w, x)$ for all $i \in\{1, \ldots, 4\}$, we conclude that $(w, x) \notin C\left(E^{M^{2}}, \bar{R}^{N}\right)$.

Observe that other methods do not perform better for the 'lexicographic best extension' in this example, by considering again Example 3.6.

\subsection{Neutrality}

An SCC is neutral if it is covariant under permutations of the alternatives. To obtain neutrality of $M$ or, more generally, $M^{k}$ one needs that all weights $\beta(x)$ are equal, but this is not always possible, due to the restriction on $\beta(\cdot)$. Of course, one can always choose the weights such that the difference between any two weights is at most one. Also, if the number of voters is large relative to the number of alternatives, then one practically obtains neutrality: e.g., if $m=10$ and $n=1000$ then one can choose nine of the weights equal to 100 and one weight equal to 101 .

Alternatively, one could take the union of the sets $M_{\beta}^{k}\left(R^{N}\right)$ over all possible weight assignments $\beta(\cdot)$. It is not clear, however, whether this enlarged neutral SCC still has the core property.

\subsection{Further literature}

Peleg and Peters (2016) provide an axiomatic characterization of the correspondence $M$. A characterization of $M^{k}$ is not yet available.

Among other recent works that are concerned with choosing committees we mention Kamwa (2014) and Laslier and Van der Straeten (2014). The former concentrates on pairwise majority relations. The latter uses approval methods; its Proposition 10 for the case of single-peaked preference profiles coincides with what feasible elimination procedures would achieve in this case.

\section{References}

Abdou, J., Keiding, H., 1991. Effectivity Functions in Social Choice. Kluwer Academic Publishers, Dordrecht.

Brams, S.J., Fishburn, P.C., 2002. Voting Procedures. Handbook of Social Choice and Welfare, vol. 1. Elsevier, Amsterdam.

Danilov, V.I., Sotskov, A., 2002. Social Choice Mechanisms. Springer Verlag, Heidelberg.

Dutta, B., Pattanaik, P.K., 1978. On nicely consistent voting systems. Econometrica 46, 163-170.

Gibbard, A., 1973. Manipulation of voting schemes: a general result. Econometrica 41, 587-602.

Holzman, R., 1986. On strong representations of games by social choice functions. J. Math. Econ. 15, 39-57.

Hopcroft, J.E., Karp, R.M., 1973. An $n^{5 / 2}$ algorithm for maximum matchings in bipartite graphs. SIAM J. Comput. 2, $225-231$.

Ishikawa, S., Nakamura, K., 1980. Representations of characteristic function games by social choice functions. Int. J. Game Theory 9, 191-199.

Kamwa, E., 2014. Essais sur les modes de scrutin et la sélection des comités. PhD thesis. Université de Caen Basse-Normandie.

Kim, K.H., Roush, F.W., 1981. Properties of consistent voting systems. Int. J. Game Theory 10, 45-52.

Laslier, J.F., Van der Straeten, K., 2014. Strategic Voting Under Committee Approval: A Theory. Toulouse School of Economics and CNRS, Paris.

Maskin, E., 1999. Nash equilibrium and welfare optimality. Rev. Econ. Stud. 66, 23-38.

Oren, I., 1981. The structure of exactly strongly consistent social choice functions. J. Math. Econ. 8, 207-220.

Peleg, B., 1978. Consistent voting systems. Econometrica 46, 153-161.

Peleg, B., 1984. Game Theoretic Analysis of Voting in Committees. Cambridge University Press, Cambridge.

Peleg, B., Peters, H., 2006. Consistent voting systems with a continuum of voters. Soc. Choice Welfare 27, 477-492.

Peleg, B., Peters, H., 2010. Strategic Social Choice. Springer, Berlin.

Peleg, B., Peters, H., 2016. Feasible elimination procedures in social choice: an axiomatic characterization. Maastricht. GSBE Research Memorandum 16/001.

Polishchuk, I., 1978. Monotonicity and uniqueness of consistent voting systems. Center for Research in Mathematical Economics and Game Theory, Hebrew University of Jerusalem.

Satterthwaite, M.A., 1975. Strategy-proofness and arrow's conditions: existence and correspondence theorems for voting procedures and social welfare functions. J. Econ. Theory 10, 187-207. 